\title{
A NEW CHARACTERIZATION OF CHORD-ARC DOMAINS
}

\author{
JONAS AZZAM, STEVE HOFMANN, JOSÉ MARÍA MARTELL, \\ KAJ NYSTRÖM, TATIANA TORO
}

\begin{abstract}
We show that if $\Omega \subset \mathbb{R}^{n+1}, n \geq 1$, is a uniform domain (aka 1sided NTA domain), i.e., a domain which enjoys interior Corkscrew and Harnack Chain conditions, then uniform rectifiability of the boundary of $\Omega$ implies the existence of exterior Corkscrew points at all scales, so that in fact, $\Omega$ is a chord-arc domain, i.e., a domain with an Ahlfors-David regular boundary which satisfies both interior and exterior Corkscrew conditions, and an interior Harnack Chain condition. We discuss some implications of this result, for theorems of $\mathrm{F}$. and M. Riesz type, and for certain free boundary problems.
\end{abstract}

\section{INTRODUCTION AND STATEMENT OF MAIN RESULT}

An NTA (Non-Tangentially Accessible) domain $\Omega$ is one which enjoys an interior Harnack Chain condition, as well as interior and exterior Corkscrew conditions (see Definitions 2.10, 2.11, and 2.16 below). This notion was introduced in [JK], and has found numerous applications in the theory of elliptic equations and free boundary problems. A chord-arc domain is an NTA domain whose boundary is Ahlfors-David regular (ADR) (see Definition 2.1 below).

A 1-sided NTA domain (also known as a uniform domain in the literature) satisfies interior Corkscrew and Harnack Chain conditions, but one imposes no assumptions on the exterior domain $\Omega_{\mathrm{ext}}:=\mathbb{R}^{n+1} \backslash \bar{\Omega}$. A 1 -sided chord-arc domain is a 1-sided NTA domain with an ADR boundary. In general, the 1-sided conditions are strictly weaker than their standard counterparts: for example, the domain $\Omega=\mathbb{R}^{2} \backslash K$, where $K$ is Garnett's "4-corners Cantor set" (see, e.g., [DS2]), is a 1 -sided chord-arc domain, but $\bar{\Omega}=\mathbb{R}^{2}$, thus, there is no exterior domain.

The various versions of non-tangential accessibility have been studied in connection with quantitative analogues of the F. and M. Riesz Theorem [RR], in which

Date: June 11, 2014.

2010 Mathematics Subject Classification. 28A75, 28A78, 31A15, 31B05, 35J25, 42B37, 49Q15.

Key words and phrases. Chord-arc domains, NTA domains, 1-sided NTA domains, uniform domains, uniform rectifiability, Carleson measures, harmonic measure, $A_{\infty}$ Muckenhoupt weights.

The first author was partially supported by NSF RTG grant 0838212 . The second author was supported by NSF grants DMS-1101244 and DMS-1361701. The third author was supported in part by MINECO Grant MTM2010-16518, ICMAT Severo Ochoa project SEV-2011-0087. He also acknowledges that the research leading to these results as received funding from the European Research Council under he European Union's Seventh Framework Programme (FP7/2007-2013)/ ERC agreement no. 615112 HAPDEGMT. The fourth author was partly supported by the Swedish research council VR. The last author was partially supported by the Robert R. \& Elaine F. Phelps Professorship in Mathematics. 
one obtains scale-invariant absolute continuity (e.g., the $A_{\infty}$ condition of Muckenhoupt, or more generally, weak- $A_{\infty}$ ) of harmonic measure $\omega$ with respect to surface measure on the boundary, given some quantitative rectifiability property of the boundary, along with some quantitative connectivity hypothesis of $\Omega$. This has been done in 2 dimensions by M. Lavrentiev [Lav]; and in higher dimensions, for Lipschitz domains by B. Dahlberg [Dah]; for chord-arc domains by G. David and D. Jerison [DJ], and independently, by S. Semmes [Sem]; for NTA domains without an ADR hypothesis by M. Badger [Bad]; for the Riesz measure associated to the $p$-Laplacian in chord-arc domains, by J. Lewis and the fourth named author of this paper [LN]; for domains with ADR boundaries, satisfying an "interior big pieces of Lipschitz sub-domains" condition, by B. Bennewitz and J. Lewis [BL]; and for 1-sided chord-arc domains with uniformly rectifiable boundaries (see Definition 2.3 below), by the second and third named authors of this paper [HM]. In addition, the second and third authors of this paper, jointly with I. Uriarte-Tuero [HMU], have obtained a free boundary result that is a converse to the theorem of $[\mathrm{HM}]$, in which scale invariant absolute continuity of harmonic measure with respect to surface measure, in the presence of the 1-sided chord-arc condition, implies uniform rectifiability of $\partial \Omega$.

It turns out, perhaps surprisingly, in light of the counter-example of T. Hrycak (see [DS2]), that the result of [HM] may be subsumed in that of [DJ] and [Sem], while on the other hand, the free boundary result of [HMU] is stronger than the authors had initially realized. Our main result in this paper is the following.

Theorem 1.1. Suppose that $\Omega \subset \mathbb{R}^{n+1}$ is a uniform (aka 1-sided NTA) domain, and that $\partial \Omega$ is uniformly rectifiable. Then $\Omega$ is a chord-arc domain.

Let us point out that both of our hypotheses are essential, in the sense that neither one, alone, allows one to draw the stated conclusion. Indeed, as we have noted above, a 1-sided NTA domain, with ADR boundary, need not be NTA in general: in fact there may be no exterior domain. On the other hand, Hrycak's example shows that uniform rectifiability, in general, is strictly weaker than a "Big Pieces of Lipschitz Graphs" condition ${ }^{1}$, whereas the latter property is enjoyed by boundaries of chord-arc domains, by the main geometric result of [DJ].

Theorem 1.1, combined with previous work mentioned above, has implications for theorems of F. and M. Riesz type, and for certain free boundary problems. We now have the following.

Theorem 1.2. Suppose that $\Omega \subset \mathbb{R}^{n+1}$ is a uniform (aka 1-sided NTA) domain, whose boundary is Ahlfors-David regular. Then the following are equivalent:

(1) $\partial \Omega$ is uniformly rectifiable.

(2) $\Omega$ is an NTA domain, and hence, a chord-arc domain.

(3) $\omega \in A_{\infty}$.

(4) $\omega \in$ weak $-A_{\infty}$.

\footnotetext{
${ }^{1}$ However, as shown by the first named author of this paper and R. Schul [AS], uniform rectifiability is equivalent to a "Big Pieces of Big Pieces of Lipschitz Graphs" condition.
} 
Here $\omega$ denotes harmonic measure for $\Omega$ with some fixed pole, and the statements (3) and (4) are understood in a scale invariant sense (see, e.g., [HM, HMU]). That (2) implies (3) was proved in [DJ], and independently, in [Sem]; it is of course trivial that (3) implies (4), while (4) implies (1) is the main result of [HMU]. Theorem 1.1 closes the circle by establishing that (1) implies (2). In particular, this yields a sharpened version of the free boundary result of [HMU], namely, we now obtain that (4) implies (2), under the stated background hypotheses.

In connection with the previous result we recall the David-Semmes conjecture recently proved in $[\mathrm{NToV}]$ (see also [HMM] for the case of a boundary of a 1-sided NTA domain with ADR boundary) which establishes that uniform rectifiability is equivalent to the boundedness of the Riesz transform. Hence Theorem 1.1 implies that, under the same background hypothesis, the Riesz transform is bounded on $L^{2}(\partial \Omega)$ if and only if $\Omega$ is an NTA domain and consequently a chord-arc domain.

In Section 2, we establish notation and review some necessary definitions and preliminary results. In particular, we recall two different characterizations of uniform rectifiability, one analytic and the other geometric. In Sections 3 and 4, we give two separate proofs of Theorem 1.1, each one using a different characterization. Finally, in an appendix, we provide the proof of a folklore theorem concerning the equivalence of 1-sided NTA and uniform domains.

\section{Preliminaries}

\subsection{Notation and conventions.}

- We use the letters $c, C$ to denote harmless positive constants, not necessarily the same at each occurrence, which depend only on dimension and the constants appearing in the hypotheses of the theorems (which we refer to as the "allowable parameters"). We shall also sometimes write $a \lesssim b$ and $a \approx b$ to mean, respectively, that $a \leq C b$ and $0<c \leq a / b \leq C$, where the constants $c$ and $C$ are as above, unless explicitly noted to the contrary. Unless otherwise specified upper case constants are greater than 1 and lower case constants are smaller than 1.

- Given a domain $\Omega \subset \mathbb{R}^{n+1}$, we shall use lower case letters $x, y$, $z$, etc., to denote points on $\partial \Omega$, and capital letters $X, Y, Z$, etc., to denote generic points in $\mathbb{R}^{n+1}$ (especially those in $\mathbb{R}^{n+1} \backslash \partial \Omega$ ).

- The open $(n+1)$-dimensional Euclidean ball of radius $r$ will be denoted $B(x, r)$ when the center $x$ lies on $\partial \Omega$, or $B(X, r)$ when the center $X \in \mathbb{R}^{n+1} \backslash \partial \Omega$. A surface ball is denoted $\Delta(x, r):=B(x, r) \cap \partial \Omega$.

- If $\partial \Omega$ is bounded, it is always understood (unless otherwise specified) that all surface balls have radii controlled by the diameter of $\partial \Omega$ : that is if $\Delta=\Delta(x, r)$ then $r \lesssim \operatorname{diam}(\partial \Omega)$. Note that in this way $\Delta=\partial \Omega$ if $\operatorname{diam}(\partial \Omega)<r \lesssim \operatorname{diam}(\partial \Omega)$.

- For $X \in \mathbb{R}^{n+1}$, we set $\delta(X):=\operatorname{dist}(X, \partial \Omega)$.

- We let $H^{n}$ denote $n$-dimensional Hausdorff measure, and let $\sigma:=\left.H^{n}\right|_{\partial \Omega}$ denote the surface measure on $\partial \Omega$. 
- For a Borel set $A \subset \mathbb{R}^{n+1}$, we let $1_{A}$ denote the usual indicator function of $A$, i.e. $1_{A}(x)=1$ if $x \in A$, and $1_{A}(x)=0$ if $x \notin A$.

- For a Borel subset $A \subset \partial \Omega$, we set $f_{A} f d \sigma:=\sigma(A)^{-1} \int_{A} f d \sigma$.

- We shall use the letter $I$ (and sometimes $J$ ) to denote a closed $(n+1)$-dimensional Euclidean cube with sides parallel to the co-ordinate axes, and we let $\ell(I)$ denote the side length of $I$. We use $Q$ to denote a dyadic "cube" on $\partial \Omega$. The latter exist, given that $\partial \Omega$ is $\mathrm{ADR}$ (cf. [DS1], [Chr]), and enjoy certain properties which we enumerate in Lemma 2.18 below.

\subsection{Some definitions.}

Definition 2.1 (Ahlfors-David regular). We say that a closed set $E \subset \mathbb{R}^{n+1}$ is $n$ dimensional ADR (or simply ADR) (Ahlfors-David regular) if there is some uniform constant $C$ such that

$$
\frac{1}{C} r^{n} \leq H^{n}(E \cap B(x, r)) \leq C r^{n}, \forall r \in\left(0, R_{0}\right), x \in E,
$$

where $R_{0}$ is the diameter of $E$ (which may be infinite).

Definition 2.3 (Uniform Rectifiability). Following David and Semmes [DS1], [DS2], we say that a closed set $E \subset \mathbb{R}^{n+1}$ is $n$-dimensional UR (or simply UR) (Uniformly Rectifiable), if it satisfies the ADR condition (2.2), and if for some uniform constant $C$ and for every Euclidean ball $B:=B\left(x_{0}, r\right), r \leq \operatorname{diam}(E)$, centered at any point $x_{0} \in E$, we have the Carleson measure estimate

$$
\iint_{B}\left|\nabla^{2} \mathcal{S} 1(X)\right|^{2} \operatorname{dist}(X, E) d X \leq C r^{n},
$$

where $\mathcal{S} f$ is the harmonic single layer potential of $f$, i.e.,

$$
\mathcal{S} f(X):=c_{n} \int_{E}|X-y|^{1-n} f(y) d H^{n}(y) .
$$

Here, the normalizing constant $c_{n}$ is chosen so that $\mathcal{E}(X):=c_{n}|X|^{1-n}$ is the usual fundamental solution for the Laplacian in $\mathbb{R}^{n+1}$ (one should use a logarithmic potential in ambient dimension $n+1=2$ ).

A geometric characterization of uniform rectifiability can be given in terms of the so called bilateral $\beta$-numbers. For $x \in E$, a hyperplane $P$, and $r>0$ we set

$$
b \beta_{E}(x, r, P)=r^{-1}\left(\sup _{y \in E \cap B(x, r)} \operatorname{dist}(y, P)+\sup _{y \in P \cap B(x, r)} \operatorname{dist}(y, E)\right)
$$

and then define

$$
b \beta_{E}(x, r)=\inf _{P} b \beta_{E}(x, r, P)
$$

where the infimum is over all $n$-dimensional hyperplanes $P \subseteq \mathbb{R}^{n+1}$.

Definition 2.6 (BWGL). We say that an $n$-dimensional ADR set $E$ satisfies the bilateral weak geometric lemma or $B W G L$ if, for each $\varepsilon>0$, the set

$$
\widehat{B}_{\varepsilon}:=\left\{(x, r): x \in E, r>0, b \beta_{E}(x, r) \geq \varepsilon\right\}
$$


is a Carleson set, i.e., there is $C_{1}>0$ so that if we define

$$
\widehat{\sigma}(A)=\iint_{A} d H^{n} \frac{d t}{t}, \quad A \subset E \times(0, \infty),
$$

then

$$
\widehat{\sigma}\left(\widehat{B}_{\varepsilon} \cap(B(x, r) \times(0, r))\right) \leq C_{1} r^{n}
$$

for all $x \in E$ and $r>0$.

Theorem 2.8 ([DS2, Theorem 2.4, Part I]). An n-dimensional ADR set $E$ is uniformly rectifiable if and only if it satisfies the BWGL.

Remark 2.9. We note that there are numerous characterizations of uniform rectifiability given in [DS1, DS2]; the two stated above will be most useful for our purposes, and appear in [DS2, Chapter 2, Part I] and [DS2, Chapter 3, Part III].

We recall that the UR sets are precisely those for which all "sufficiently nice" singular integrals are bounded on $L^{2}$ (see [DS1]). We further remark that uniform rectifiability is the scale invariant version of rectifiability: in particular, BWGL may be viewed as a quantitative version of the characterization of rectifiable sets in terms of the existence a.e. of approximate tangent planes. In this context, see also [Jo1].

Definition 2.10 (Corkscrew condition). Following [JK], we say that a domain $\Omega \subset \mathbb{R}^{n+1}$ satisfies the Corkscrew condition if for some uniform constant $c>0$ and for every surface ball $\Delta:=\Delta(x, r)$, with $x \in \partial \Omega$ and $0<r<\operatorname{diam}(\partial \Omega)$, there is a ball $B\left(X_{\Delta}, c r\right) \subset B(x, r) \cap \Omega$. The point $X_{\Delta} \subset \Omega$ is called a corkscrew point relative to $\Delta$, (or, relative to $B$ ). We note that we may allow $r<C \operatorname{diam}(\partial \Omega)$ for any fixed $C$, simply by adjusting the constant $c$.

Definition 2.11 (Harnack Chain condition). Again following [JK], we say that $\Omega$ satisfies the Harnack Chain condition if there is a uniform constant $C$ such that for every $\rho>0, \Lambda \geq 1$, and every pair of points $X, X^{\prime} \in \Omega$ with $\delta(X), \delta\left(X^{\prime}\right) \geq \rho$ and $\left|X-X^{\prime}\right|<\Lambda \rho$, there is a chain of open balls $B_{1}, \ldots, B_{N} \subset \Omega, N \leq C(\Lambda)$, with $X \in B_{1}, X^{\prime} \in B_{N}, B_{k} \cap B_{k+1} \neq \varnothing$ and $C^{-1} \operatorname{diam}\left(B_{k}\right) \leq \operatorname{dist}\left(B_{k}, \partial \Omega\right) \leq C \operatorname{diam}\left(B_{k}\right)$. The chain of balls is called a Harnack Chain.

Definition 2.12 (1-sided NTA). If $\Omega$ satisfies both the Corkscrew and Harnack Chain conditions, then we say that $\Omega$ is a 1-sided NTA domain.

Remark 2.13. We observe that the 1-sided NTA condition is a quantitative connectivity condition.

An alternative (and quantitatively equivalent) definition is as follows.

Definition 2.14 (Uniform domain). For $0<c<1<C$, we say that an open set $\Omega \subseteq \mathbb{R}^{n+1}$ is a $(c, C)$-uniform domain if, for any two points $X, Y \in \Omega$, there is a path $\gamma$ so that

(1) $\ell(\gamma) \leq C|X-Y|$, where $\ell(\gamma)$ denotes the length of $\gamma$, and

(2) for any $Z \in \gamma, \delta(Z) \geq c \operatorname{dist}(Z,\{X, Y\})$.

We call such a curve a good curve for $X$ and $Y$. 
It is well known that Definitions 2.12 and 2.14 are equivalent, nevertheless finding a precise reference is difficult. That every uniform domain is a 1-sided NTA domain was proved by Gehring and Osgood [GO]. Indeed, the extension domains of P. Jones [Jo2] are clearly 1-sided NTA by definition, while in [GO], the authors prove that extension domains are the same as uniform domains (see also [Väi]). For a more direct proof of the fact that uniform domains are 1-sided NTA domains, see [BS, Lemma 4.2 and 4.3], as well as their proofs. For completeness, we include the converse implication below.

Theorem 2.15. If $\Omega$ is a 1-sided NTA domain then $\Omega$ is a uniform domain with constants that only depend on the 1-sided NTA constants.

We defer the proof of Theorem 2.15 to an appendix.

Definition 2.16 (NTA domain). Following [JK], we say that a domain $\Omega$ is an NTA domain if it is a 1-sided NTA domain and if, in addition, $\Omega_{\mathrm{ext}}:=\mathbb{R}^{n+1} \backslash \bar{\Omega}$ also satisfies the Corkscrew condition.

Definition 2.17 (Chord-arc domain). $\Omega$ is a chord-arc domain if it is an NTA domain with an ADR boundary.

\subsection{Dyadic grids.}

Lemma 2.18 (Existence and properties of the "dyadic grid"). [DS1, DS2], [Chr]. Suppose that $E \subset \mathbb{R}^{n+1}$ satisfies the ADR condition (2.2). Then there exist constants $a_{0}>0, \eta>0$ and $C_{1}<\infty$, depending only on dimension and the ADR constants, such that for each $k \in \mathbb{Z}$, there is a collection of Borel sets ("cubes")

$$
\mathbb{D}_{k}:=\left\{Q_{j}^{k} \subset E: j \in \mathfrak{J}_{k}\right\}
$$

where $\mathfrak{I}_{k}$ denotes some (possibly finite) index set depending on $k$, satisfying

(i) $E=\cup_{j} Q_{j}^{k}$ for each $k \in \mathbb{Z}$.

(ii) If $m \geq k$ then either $Q_{i}^{m} \subset Q_{j}^{k}$ or $Q_{i}^{m} \cap Q_{j}^{k}=\varnothing$.

(iii) For each $(j, k)$ and each $m<k$, there is a unique i such that $Q_{j}^{k} \subset Q_{i}^{m}$.

(iv) Diameter $\left(Q_{j}^{k}\right) \leq C_{1} 2^{-k}$.

(v) Each $Q_{j}^{k}$ contains some surface ball $\Delta\left(x_{j}^{k}, a_{0} 2^{-k}\right):=B\left(x_{j}^{k}, a_{0} 2^{-k}\right) \cap E$.

(vi) $H^{n}\left(\left\{x \in Q_{j}^{k}: \operatorname{dist}\left(x, E \backslash Q_{j}^{k}\right) \leq \tau 2^{-k}\right\}\right) \leq C_{1} \tau^{\eta} H^{n}\left(Q_{j}^{k}\right)$, for all $k, j$ and for all $\tau \in\left(0, a_{0}\right)$.

A few remarks are in order concerning this lemma.

- In the setting of a general space of homogeneous type, this lemma has been proved by Christ [Chr], with the dyadic parameter $1 / 2$ replaced by some constant $\delta \in(0,1)$. In fact, one may always take $\delta=1 / 2$ (cf. [HMMM, Proof of Proposition 2.12]). In the presence of the Ahlfors-David property (2.2), the result already appears in [DS1, DS2].

- For our purposes, we may ignore those $k \in \mathbb{Z}$ such that $2^{-k} \gtrsim \operatorname{diam}(E)$, in the case that the latter is finite. 
- We shall denote by $\mathbb{D}=\mathbb{D}(E)$ the collection of all relevant $Q_{j}^{k}$, i.e.,

$$
\mathbb{D}:=\cup_{k} \mathbb{D}_{k},
$$

where, if $\operatorname{diam}(E)$ is finite, the union runs over those $k$ such that $2^{-k} \lesssim \operatorname{diam}(E)$.

- Given a cube $Q \in \mathbb{D}$, we set

$$
\mathbb{D}_{Q}:=\left\{Q^{\prime} \in \mathbb{D}: Q^{\prime} \subseteq Q\right\},
$$

- For a dyadic cube $Q \in \mathbb{D}_{k}$, we shall set $\ell(Q)=2^{-k}$, and we shall refer to this quantity as the "length" of $Q$. Evidently, $\ell(Q) \approx \operatorname{diam}(Q)$.

- Properties (iv) and (v) imply that for each cube $Q \in \mathbb{D}_{k}$, there is a point $x_{Q} \in E$, a Euclidean ball $B\left(x_{Q}, r_{Q}\right)$ and a surface ball $\Delta\left(x_{Q}, r_{Q}\right):=B\left(x_{Q}, r_{Q}\right) \cap E$ such that $c \ell(Q) \leq r_{Q} \leq \ell(Q)$, for some uniform constant $c>0$, and

$$
\Delta\left(x_{Q}, 2 r_{Q}\right) \subset Q \subset \Delta\left(x_{Q}, C r_{Q}\right),
$$

for some uniform constant $C$. We shall denote this ball and surface ball by

$$
B_{Q}:=B\left(x_{Q}, r_{Q}\right), \quad \Delta_{Q}:=\Delta\left(x_{Q}, r_{Q}\right),
$$

and we shall refer to the point $x_{Q}$ as the "center" of $Q$.

It will be useful to dyadicize the Corkscrew condition, and to specify precise Corkscrew constants. Let us now specialize to the case that $E=\partial \Omega$ is ADR, with $\Omega$ satisfying the Corkscrew condition. Given $Q \in \mathbb{D}(\partial \Omega)$, we shall sometimes refer to a "Corkscrew point relative to $Q$ ", which we denote by $X_{Q}$, and which we define to be the corkscrew point $X_{\Delta}$ relative to the surface ball $\Delta:=\Delta_{Q}$ (see (2.20), (2.21) and Definition 2.10). We note that

$$
\delta\left(X_{Q}\right) \approx \operatorname{dist}\left(X_{Q}, Q\right) \approx \operatorname{diam}(Q) .
$$

Definition 2.23. ( $c_{0}$-exterior Corkscrew condition). Fix a constant $c_{0} \in(0,1)$, and a domain $\Omega \subset \mathbb{R}^{n+1}$, with ADR boundary. We say that a cube $Q \in \mathbb{D}(\partial \Omega)$ satisfies the the $c_{0}$-exterior Corkscrew condition, if there is a point $z_{Q} \in \Delta_{Q}$, and a point $X_{Q}^{-} \in B\left(z_{Q}, r_{Q} / 4\right) \backslash \bar{\Omega}$, such that $B\left(X_{Q}^{-}, c_{0} \ell(Q)\right) \subset B\left(z_{Q}, r_{Q} / 4\right) \backslash \bar{\Omega}$, where $\Delta_{Q}=\Delta\left(x_{Q}, r_{Q}\right)$ is the surface ball defined above in (2.20)-(2.21).

\section{The ANalytic Proof of TheORem 1.1}

We suppose that $\Omega$ is a 1 -sided NTA (i.e., uniform) domain, with uniformly rectifiable boundary. It suffices to show that $\Omega$ satisfies an exterior Corkscrew condition at all scales (up to the diameter of $\partial \Omega$ ).

To this end, we define a discrete measure $\mathrm{m}$ as follows. Let $\mathcal{B}=\mathcal{B}\left(c_{0}\right)$ denote the collection of $Q \in \mathbb{D}(\partial \Omega)$ for which the $c_{0}$-exterior Corkscrew condition (see Definition 2.23) fails. Set

$$
\alpha_{Q}:= \begin{cases}\sigma(Q), & \text { if } Q \in \mathcal{B}, \\ 0, & \text { otherwise } .\end{cases}
$$

For any subcollection $\mathbb{D}^{\prime} \subset \mathbb{D}(\partial \Omega)$, we set

$$
\mathfrak{m}\left(\mathbb{D}^{\prime}\right):=\sum_{Q \in \mathbb{D}^{\prime}} \alpha_{Q}
$$


We will prove, as a consequence of the UR and 1-sided NTA properties, that the collection $\mathcal{B}$ satisfies a packing condition, i.e., that $m$ is a discrete Carleson measure, provided that $c_{0}$ is small enough. More precisely, we have the following.

Lemma 3.3. Let $\Omega$ be a 1-sided NTA domain with UR boundary, and let $\mathcal{B}=$ $\mathcal{B}\left(c_{0}\right) \subset \mathbb{D}$ be the collection defined above. Then there is a $c_{0}$ sufficiently small, such that the measure $m$ satisfies the packing condition

$$
\sup _{Q \in \mathbb{D}} \frac{\mathfrak{m}\left(\mathbb{D}_{Q}\right)}{\sigma(Q)}=\sup _{Q \in \mathbb{D}} \frac{1}{\sigma(Q)} \sum_{Q^{\prime} \in \mathcal{B}: Q^{\prime} \subset Q} \sigma\left(Q^{\prime}\right) \leq M_{1},
$$

where the constants $c_{0}$ and $M_{1}$ depend only upon dimension, and on the $A D R / U R$ and 1-sided NTA constants.

Let us momentarily take the lemma for granted, and deduce the conclusion of Theorem 1.1. We fix a cube $Q \in \mathbb{D}(\partial \Omega)$, and we seek to show that $\Omega_{\text {ext }}$ has a Corkscrew point relative to $Q$. Let $\Delta_{Q} \subset Q$ denote the surface ball defined in (2.20)-(2.21), and let $Q_{1}$ be a sub-cube of $Q$, of maximal size, that is contained in $\Delta_{Q}$. We then have that $\ell\left(Q_{1}\right) \geq c \ell(Q)$. By (3.4) (applied to $Q_{1}$ ), and the ADR condition, there is a constant $c_{1}$, depending only on $M_{1}$ and the ADR constants, and a cube $Q^{\prime} \in \mathbb{D}_{Q_{1}} \backslash \mathcal{B}$, with $\ell\left(Q^{\prime}\right) \geq c_{1} \ell(Q)$. Since $Q^{\prime} \notin \mathcal{B}$, it therefore enjoys the $c_{0}$-exterior Corkscrew condition, and therefore, so does $Q$, but with $c_{0}$ replaced by $c_{0}^{\prime}=c_{0} c_{1}$. Since every surface ball contain a cube of comparable diameter, this means that there is an exterior Corkscrew point relative to every surface ball on the boundary, and therefore $\Omega$ is NTA, and hence chord-arc.

It remains to prove the lemma.

Proof of Lemma 3.3. We follow a related argument in [HM], which in turn uses an idea from [DS2]. Let $\mathcal{B}$ denote the collection of $Q \in \mathbb{D}$ for which the $c_{0}$-exterior Corkscrew condition (cf. Definition 2.23) fails. We fix a cube $Q \in \mathcal{B}$, a point $z_{Q} \in \Delta_{Q} \subset Q$, and set $B=B\left(z_{Q}, r / 4\right)$, with $r=r_{Q} \approx \ell(Q)$, and set $\Delta=B \cap \partial \Omega$. Let $\Phi \in C_{0}^{\infty}(B)$, with $0 \leq \Phi \leq 1, \Phi \equiv 1$ on $(1 / 2) B$, and $\|\nabla \Phi\|_{\infty} \lesssim r^{-1}$. Let $\mathcal{L}:=-\operatorname{div} \cdot \nabla$ denote the usual Laplacian in $\mathbb{R}^{n+1}$, and let $\mathcal{S}$ denote the single layer potential for $\mathcal{L}$, as in (2.5) with $E=\partial \Omega$. Since $\partial \Omega$ is ADR,

$$
\begin{array}{r}
\sigma(\Delta) \approx \int_{\partial \Omega} \Phi d \sigma=\langle\mathcal{L} \mathcal{S} 1, \Phi\rangle=\iint_{\mathbb{R}^{n+1}}(\nabla \mathcal{S} 1(X)-\vec{\beta}) \cdot \nabla \Phi(X) d X \\
\lesssim \frac{1}{r}\left(\iint_{B \backslash \Omega}|\nabla \mathcal{S} 1(X)-\vec{\beta}| d X+\iint_{B \cap \Omega}|\nabla \mathcal{S} 1(X)-\vec{\beta}| d X\right) \\
=: \frac{1}{r}(I+I I),
\end{array}
$$

where $\vec{\beta}$ is a constant vector at our disposal. We let $B^{*}:=\kappa_{0} B$ be a concentric dilate of $B$ with $\kappa_{0}$ a large constant (see [HM, (5.12)]), and set $\Delta^{*}:=B^{*} \cap \partial \Omega$. As in the proof of [HM, Lemma 5.10], we may choose $\vec{\beta}$ so that the following properties hold:

$$
\left\|\nabla S 1_{\partial \Omega \backslash \Delta^{*}}-\vec{\beta}\right\|_{L^{\infty}(B)} \leq C,
$$

and 


$$
\begin{aligned}
r^{-1} \iint_{B \cap\left(\Omega \mid \Sigma_{B, \varepsilon}\right)}|\nabla \mathcal{S} 1(X)-\vec{\beta}| & \\
& \leq C_{\varepsilon} r^{n / 2}\left(\iint_{U_{B, \varepsilon}}\left|\nabla^{2} \mathcal{S} 1(X)\right|^{2} \delta(X) d X\right)^{1 / 2},
\end{aligned}
$$

where $\Sigma_{B, \varepsilon}$ is a "border strip" of thickness $C \varepsilon r$, and $U_{B, \varepsilon}$ is a Whitney region with $\varepsilon r \lesssim \delta(X) \approx \operatorname{dist}(X, \Delta) \lesssim r$, for all $X \in U_{B, \varepsilon}$, with $\varepsilon$ a small positive constant to be chosen. In the language of [HM, Sections 4 and 5], $\Sigma_{B, \varepsilon}=\Omega \backslash \Omega_{\mathcal{F}(\epsilon r), Q}$ with $\mathcal{F}=\varnothing, U_{B, \varepsilon}=\Omega_{\mathcal{F}(\epsilon r), Q}^{\text {fat }}$ and $\vec{\beta}$ is the average of $\nabla S 1$ on $\Omega_{\mathcal{F}(\epsilon r), Q \text {. We observe }}$ that (3.7) is a consequence of a Poincare inequality proved in [HM, Section 4]. Moreover, by [HM, Lemma 5.1, Lemma 5.3, and Corollary 5.6], we have

$$
\begin{gathered}
\iint_{B}\left|\nabla \mathcal{S} 1_{\Delta^{*}}(X)\right|^{q} d X \leq C_{q} r^{n+1}, \quad 1 \leq q<(n+1) / n, \\
\left|\Sigma_{B, \varepsilon} \cap B\right| \lesssim \varepsilon r^{n+1},
\end{gathered}
$$

and

$$
\iint_{B \cap \Sigma_{B, \varepsilon}}\left|\nabla \mathcal{S} 1_{\Delta^{*}}(X)\right| d X \lesssim \varepsilon^{\gamma} r^{n+1},
$$

for some fixed $\gamma$, with $0<\gamma<1$. Combining these facts, we see that

$$
\frac{I I}{r} \lesssim \varepsilon^{\gamma} \sigma(\Delta)+C_{\varepsilon} \sigma(\Delta)^{1 / 2}\left(\iint_{U_{B, \varepsilon}}\left|\nabla^{2} \mathcal{S} 1(X)\right|^{2} \delta(X) d X\right)^{1 / 2} .
$$

Furthermore, by [HM, Lemma 5.7], the failure of the $c_{0}$-exterior Corkscrew property implies that

$$
|B \backslash \Omega| \lesssim c_{0} r^{n+1} .
$$

Combining the latter fact with (3.6) and (3.8), we find that

$$
\frac{I}{r} \lesssim c_{0}^{1 / q^{\prime}} \sigma(\Delta) \text {. }
$$

If $\varepsilon$ and $c_{0}$ are chosen small enough, then the small terms may be hidden on the left hand side of (3.5), to obtain that

$$
\sigma(\Delta) \lesssim \iint_{U_{B, \varepsilon}}\left|\nabla^{2} \mathcal{S} 1(X)\right|^{2} \delta(X) d X .
$$

As observed above, the measure $d m(X):=\left|\nabla^{2} \mathcal{S} 1(X)\right|^{2} \delta(X) d X$ is a Carleson measure in $\mathbb{R}^{n+1} \backslash \partial \Omega$, since $\partial \Omega$ is UR (see (2.4)). Moreover, for the various balls $B=B\left(z_{Q}, r / 4\right)$ under consideration, the Whitney regions $U_{B, \varepsilon}$ have bounded overlaps, since each such region is associated to a cube $Q$ with $\ell(Q) \approx r$, and $B \cap \partial \Omega \subset Q$ (in the language of [HM, Sections 3 and 4], $U_{B, \varepsilon}$ is a union of fattened Whitney boxes meeting $B^{*}$ whose side length is of the order of $\left.\ell(Q)\right)$. In addition,

$$
\sigma(Q) \lesssim \sigma(\Delta) .
$$

Combining these observations with (3.11), we obtain the desired packing condition. 


\section{THE GeOMETRIC PROOF OF THEOREM 1.1}

Suppose $\Omega$ is a $(c, C)$-uniform domain with ADR boundary $E:=\partial \Omega$. As mentioned earlier, this is equivalent to being 1-sided NTA, so in particular, we will also use the fact that $\Omega$ satisfies the corkscrew condition in Definition 2.10. We can assume, by making numbers smaller if need it, that the constant $c$ in the definition of the corkscrew condition is the same value as the $c$ in our definition of $(c, C)$-uniform domains.

Lemma 4.1. There is $\varepsilon>0$ depending only on $c$ and $C$ such that the following holds. Suppose $x \in \partial \Omega, r \in(0, \operatorname{diam}(\partial \Omega))$, and that $P$ is a hyperplane such that $b \beta(x, r, P)<\varepsilon$. Let $v_{P}$ be a unit vector orthogonal to $P$. Define

$$
B^{ \pm}(x, r)=B(x, r) \cap\left\{x+y: \pm y \cdot v_{P}>\varepsilon r\right\} \subseteq \mathbb{R}^{n+1} \backslash \partial \Omega .
$$

Then exactly one of either $B^{ \pm}(x, r)$ is contained in $\Omega_{\mathrm{ext}}$ and the other one is contained in $\Omega$.

We assume Lemma 4.1 for the moment and complete the proof of Theorem 1.1. Fix $\varepsilon>0$ as in Lemma 4.1 and $B=B(x, r)$ with $x \in E$ and $r<\operatorname{diam}(\partial \Omega)$. Our aim is to show that $B$ contains an exterior corkscrew. Set $(1 / 2) \Delta=\Delta(x, r / 2)$. By Definition 2.6 and Theorem 2.8, there is $C_{1}$ (depending on $\varepsilon$ ) so that (2.7) holds. If we set

$$
\rho=\sup \left\{s<r / 2: \exists y \in(1 / 2) \Delta,(y, s) \notin \widehat{B}_{\varepsilon}\right\},
$$

then

$$
\begin{aligned}
C_{1} \frac{r^{n}}{2^{n}} \geq \widehat{\sigma}\left(\widehat{B}_{\varepsilon} \cap(1 / 2) \Delta \times(0, r / 2)\right) \geq & \widehat{\sigma}((1 / 2) \Delta \times(\rho, r / 2)) \\
& =\sigma((1 / 2) \Delta) \log \frac{r}{2 \rho} \geq C^{-1} \frac{r^{n}}{2^{n}} \log \frac{r}{2 \rho},
\end{aligned}
$$

where $C^{-1}$ is the constant from (2.2). Thus $\rho \approx r$ (with implicit constants depending on $\varepsilon$ and $n$ ), hence we may find $x_{1} \in(1 / 2) \Delta$ and $r \lesssim r_{1} \leq r / 2$ so that $B\left(x_{1}, r_{1}\right) \subseteq B(x, r)$ and $b \beta\left(x_{1}, r_{1}\right)<\varepsilon$. Lemma 4.1 and the fact that $r_{1} \approx r$ imply $B$ has an exterior corkscrew, and this finishes the proof of Theorem 1.1.

Next we prove Lemma 4.1.

Proof of Lemma 4.1. Let $k=1 /(2 C+1), X^{ \pm}=x \pm k r v_{P} / 2$, and $\varepsilon<c k / 4$, so that $X^{ \pm} \in \mathbb{R}^{n+1} \backslash \partial \Omega$. We will often use the fact that $k<1$.

Claim: At least one of $X^{ \pm}$is in $\Omega_{\mathrm{ext}}$. Indeed, assume on the contrary that both points are contained in $\Omega$. Then there is a good curve $\gamma \subseteq \Omega$ connecting $X^{ \pm}$such that

$$
\operatorname{diam}(\gamma) \leq \ell(\gamma) \leq C\left|X^{+}-X^{-}\right|=C k r .
$$

By our choice of $k$, and since $X^{+} \in \gamma$, this implies that

$$
\gamma \subseteq B\left(X^{+}, C k r\right) \subseteq B(x, k r(C+1 / 2)) \subseteq B(x, r) .
$$

Furthermore, by condition (2) in Definition 2.14 there must exist $Z \in \gamma \cap P \cap B(x, r)$, such that

$$
\min \left\{\left|Z-X^{+}\right|,\left|Z-X^{-}\right|\right\} \leq \frac{\delta(Z)}{c} \leq \frac{\varepsilon r}{c} .
$$


Assume $\left|Z-X^{+}\right| \leq\left|Z-X^{-}\right|$and let $x^{\prime}$ denote the orthogonal projection of $x$ onto $L$. Then

$$
\frac{\varepsilon r}{c} \geq\left|X^{+}-Z\right| \geq\left|X^{+}-x^{\prime}\right| \geq\left|X^{+}-x\right|-\left|x-x^{\prime}\right| \geq \frac{k r}{2}-\varepsilon r>\frac{k r}{4},
$$

which is a contradiction since $\varepsilon<\frac{c k}{4}$. This proves the claim.

We have proved that either $X^{+}$or $X^{-}$belong to $\Omega_{\text {ext }}$. Suppose for instance that $X^{-} \in \Omega_{\mathrm{ext}}$. Note that $B^{-}(x, r) \subseteq \Omega_{\mathrm{ext}}$ since $B^{-}(x, r)$ it is connected, contains $X^{-}$, and does not intersect $\partial \Omega$ (because $b \beta(x, r)<\varepsilon$ ). Similarly $B^{+}(x, r) \cap \partial \Omega=\varnothing$. To show that $B^{+}(x, r)$ is contained in $\Omega$, we recall that, by the corkscrew condition, there is $X_{\Delta} \in B(x, r) \cap \Omega$ such that $B\left(X_{\Delta}, c r\right) \subseteq B(x, r) \cap \Omega$. Since $\varepsilon<c k / 4<c / 4$, we know that $B\left(X_{\Delta}, c r\right)$ must intersect either $B^{+}(x, r)$ or $B^{-}(x, r)$. Since it is contained in $\Omega$, it cannot hit $B^{-}(x, r)$, thus it must intersect $B^{+}(x, r)$. Since $B^{+}(x, r)$ is connected, $B^{+}(x, r)$ must also be contained in $\Omega$ and this completes the proof. Note that, as far as Theorem 1.1 is concerned, what is more relevant to us is that $B^{-}(x, r) \subseteq \Omega_{\mathrm{ext}}$, but we have shown $B^{+}(x, r) \subseteq \Omega$ for the sake of completeness. A similar argument appears in the proof of Theorem 1.18 [Dav].

\section{Appendix A. Proof of Theorem 2.15}

Lemma A.1. Let $X, X^{\prime} \in \Omega, \rho:=\min \left\{\delta(X), \delta\left(X^{\prime}\right)\right\}$, and $\left|X-X^{\prime}\right| \leq \Lambda \rho$. Let $B_{1}, \ldots, B_{N}$ with $N \leq C(\Lambda)$ be a Harnack chain with $X \in B_{1}, X^{\prime} \in B_{N}, B_{k} \cap B_{k+1} \neq \varnothing$ and $C_{0}^{-1} \operatorname{diam}\left(B_{k}\right) \leq \operatorname{dist}\left(B_{k}, \partial \Omega\right) \leq C_{0} \operatorname{diam}\left(B_{k}\right)$. Then $\operatorname{diam}\left(B_{j}\right) \approx \rho$ with constants depending only on $\Lambda$ and $C_{0}$. Let $\gamma$ denote the polygonal curve connecting $X$ to the center of $B_{1}$, then the centers of the $B_{j}$ 's in order and then the center of $B_{N}$ to $X^{\prime}$. Then $\ell(\gamma) \leq M\left|X-X^{\prime}\right|$ where $M$ depends only on $n, \Lambda$ and $C_{0}$. Moreover for any $Z \in \gamma$ it follows that $\delta(Z) \geq c \operatorname{dist}\left(Z,\left\{X, X^{\prime}\right\}\right)$ for some $c$ that only depends on $n, \Lambda$ and $C_{0}$. Thus $\gamma$ is a good curve for $X$ and $X^{\prime}$.

Proof. Note that if $\left|X-X^{\prime}\right| \leq \rho / 2$ then the Harnack chain above can be taken to only have one ball and the segment joining $X$ to $X^{\prime}$ is a good curve. Thus we assume $\left|X-X^{\prime}\right| \geq \rho / 2$. Since $B_{j} \cap B_{j+1} \neq \varnothing$ for $j<N$ then

$$
\begin{aligned}
\operatorname{diam}\left(B_{j}\right) \leq C_{0} \operatorname{dist}\left(B_{j}, \partial \Omega\right) \leq C_{0}\left(\operatorname{dist}\left(B_{j+1}, \partial \Omega\right)+\right. & \left.\operatorname{diam}\left(B_{j+1}\right)\right) \\
& \leq C_{0}\left(C_{0}+1\right) \operatorname{diam}\left(B_{j+1}\right),
\end{aligned}
$$

and switching the $\operatorname{roles} \operatorname{diam}\left(B_{j+1}\right) \leq C_{0}\left(C_{0}+1\right) \operatorname{diam}\left(B_{j}\right)$. Thus for $j=1, \ldots, N$, $\operatorname{diam}\left(B_{j}\right) \approx \min \left\{\operatorname{diam}\left(B_{1}\right), \operatorname{diam}\left(B_{N}\right)\right\} \approx \rho$ with comparability constants depending only on $n, \Lambda$ and $C_{0}$. Note that if $\left|X-X^{\prime}\right| \geq \rho / 2$ then

$$
\ell(\gamma) \leq \sum_{i=1}^{N} \operatorname{diam}\left(B_{i}\right)+\operatorname{diam}\left(B_{1}\right)+\operatorname{diam}\left(B_{N}\right) \lesssim N \rho \leq C(\Lambda) \rho \lesssim\left|X-X^{\prime}\right| .
$$

If $Z \in \gamma$ then there is $i=1, \ldots, N$ such that $Z \in B_{i}$. Assume for instance that $\operatorname{dist}\left(Z,\left\{X, X^{\prime}\right\}\right)=|Z-X|$, then

$$
\operatorname{dist}\left(Z,\left\{X, X^{\prime}\right\}\right) \leq \operatorname{diam}\left(B_{1}\right)+\sum_{j=1}^{i} \operatorname{diam}\left(B_{j}\right) \lesssim \rho \lesssim \operatorname{dist}\left(B_{i}, \partial \Omega\right) \lesssim \delta(Z) .
$$

This eventually shows that $\gamma$ is good curve for $X$ and $X^{\prime}$ completing the proof. 
Proof of Theorem 2.15. For $X, Y \in \Omega$ we need to find a good curve $\gamma$ connecting $X$ and $Y$. Let $k \in \mathbb{Z}$ satisfy $2^{k} \leq|X-Y|<2^{k+1}$. Let $j_{X}, j_{Y} \in \mathbb{Z}$ be such that $2^{j_{X}} \leq \delta(X)<2^{j_{X}+1}$ and $2^{j_{Y}} \leq \delta(Y)<2^{j_{Y}+1}$. Let $q_{X}, q_{Y} \in \partial \Omega$ denote respectively a closest point to $X$ and $Y$ in the boundary, that is, $\delta(X)=\left|q_{X}-X\right|$ and $\delta(Y)=\left|q_{Y}-Y\right|$.

We consider several cases.

Case 1: $|X-Y| \leq 1 / 2 \min \{\delta(X), \delta(Y)\}$ then the segment joining $X$ to $Y$ is a good curve.

Case 2: $|X-Y| \geq 1 / 2 \min \{\delta(X), \delta(Y)\}$ and $k \leq \min \left\{j_{X}+2, j_{Y}+2\right\}$ then $|X-Y|<$ $2^{k+1} \leq 8 \min \{\delta(X), \delta(Y)\}$. By Lemma A.1 there is a good curve $\gamma$ joining $X$ to $Y$.

Case 3: $|X-Y| \geq 1 / 2 \min \{\delta(X), \delta(Y)\}$ and $k \geq \min \left\{j_{X}+2, j_{Y}+2\right\}$. Switching $X$ and $Y$ we may assume that $j_{Y}+2 \geq j_{X}+2$ and therefore $k \geq j_{X}+2$. For all $i \in \mathbb{Z}$ with $j_{X}+2 \leq i \leq k$, let $X_{i}$ be a corkscrew point relative to $B\left(q_{X}, 2^{i}\right) \cap \partial \Omega$ so that $B\left(X_{i}, c 2^{i}\right) \subseteq B\left(q_{X}, 2^{i}\right)$. Provided $c \leq 1 / 4, X_{j_{X}+2}$ can be chosen to be $X$.

Note that $\left|X_{i}-X_{i+1}\right| \leq 2^{i+2}$ while $\min \left\{\delta\left(X_{i}\right), \delta\left(X_{i+1}\right)\right\} \geq c 2^{i}$, thus there is a Harnack chain $B_{1}^{i}, \ldots, B_{N_{i}}^{i}$ with $N_{i} \leq C(4 / c)$ which joins $X_{i}$ and $X_{i+1}$, and so that $X_{i} \in B_{1}^{i}$ and $X_{i+1} \in B_{N_{i}}^{i}$. Let $\gamma_{i}$ be the polygonal curve connecting $X_{i}$ to $X_{i+1}$ as constructed in Lemma A.1. Note that $\ell\left(\gamma_{i}\right) \lesssim\left|X_{i}-X_{i+1}\right| \lesssim 2^{i}$. Let $\gamma_{X}=\cup_{i=j_{X}+2}^{k-1} \gamma_{i}$. Note that $\gamma_{X}$ joins $X$ to $X_{k}$ and

$$
\ell\left(\gamma_{X}\right) \leq \sum_{i=j_{X}+2}^{k-1} \ell\left(\gamma_{i}\right) \lesssim \sum_{i=j_{X}+2}^{k-1}\left|X_{i}-X_{i+1}\right| \lesssim \sum_{i=j_{X}+2}^{k-1} 2^{i} \lesssim 2^{k} \leq|X-Y| .
$$

Moreover, note that if $Z \in \gamma_{X}$, then $Z \in \gamma_{i}$ for some $i=j_{X}+2, \ldots, k-1$. By Lemma A.1 and the construction of $\gamma_{i}$ we have that $\delta(Z) \geq c \operatorname{dist}\left(Z,\left\{X_{i}, X_{i+1}\right\}\right)$. We may assume $\operatorname{dist}\left(Z,\left\{X_{i}, X_{i+1}\right\}\right)=\left|Z-X_{i}\right|$ (the other case is treated similarly), then as in (A.2) (here $\rho \approx 2^{i}$ ) we have that $2^{i} \lesssim \delta(Z)$, which yields

$$
\begin{aligned}
|Z-X| \leq\left|Z-X_{i}\right|+\left|X_{i}-q_{X}\right|+\left|q_{X}-X\right| \leq \frac{1}{c} \delta(Z) & +2^{i}+\delta(X) \\
& \lesssim \delta(Z)+2^{j_{X}+1} \lesssim \delta(Z) .
\end{aligned}
$$

To proceed we next observe that

$$
2^{j_{Y}} \leq \delta(Y) \leq|Y-X|+\delta(X) \leq 2^{k+1}+2^{j_{X}+1} \leq 2^{k+1}+2^{k-1},
$$

which implies that $j_{Y} \leq k+1$. Assume first that $j_{Y}+2 \leq k$ (the case $k+1 \leq j_{Y}+2 \leq$ $k+3$ is considered below). Repeating the previous argument we can find a curve $\gamma_{Y}$ joining $Y$ and $Y_{k}$, where $Y_{k}$ is a corkscrew point relative to $B\left(q_{Y}, 2^{k}\right) \cap \Omega$ so that $B\left(Y_{k}, c 2^{k}\right) \subseteq B\left(q_{Y}, 2^{k}\right)$. This construction gives much as before $\ell\left(\gamma_{Y}\right) \lesssim|X-Y|$ and $|Z-Y| \lesssim \delta(Z)$ for every $Z \in \gamma_{Y}$.

We also observe that

$$
\begin{aligned}
&\left|X_{k}-Y_{k}\right| \leq\left|X_{k}-q_{X}\right|+\left|q_{X}-X\right|+|X-Y|+\left|Y-q_{Y}\right|+\left|q_{Y}-Y_{k}\right| \\
& \leq 2^{k}+2^{j_{X}+1}+2^{k+1}+2^{j_{Y}+1}+2^{k} \leq 2^{k+3} \leq 8|X-Y|
\end{aligned}
$$

and

$$
\min \left\{\delta\left(X_{k}\right), \delta\left(Y_{k}\right)\right\} \geq c 2^{k}
$$


Applying Lemma A.1 there exists a good curve $\gamma_{k}$ joining $X_{k}$ and $Y_{k}$. Let $\gamma=$ $\gamma_{X} \cup \gamma_{k} \cup \gamma_{Y}$, and note that $\gamma$ joins $X$ and $Y$. Moreover, since $2^{k} \leq|X-Y|<2^{k+1}$ then

$$
\ell(\gamma) \leq \ell\left(\gamma_{X}\right)+\ell\left(\gamma_{k}\right)+\ell\left(\gamma_{Y}\right) \lesssim|X-Y|+\left|X_{k}-Y_{k}\right| \lesssim|X-Y| .
$$

For $Z \in \gamma_{X} \cup \gamma_{Y}$, (A.3) and its corresponding version for $Y$ show that $\delta(Z) \geq$ $c \operatorname{dist}(Z,\{X, Y\})$. If $Z \in \gamma_{k}$, assume for instance that $\operatorname{dist}\left(Z,\left\{X_{k}, Y_{k}\right\}\right)=\left|X_{k}-Z\right|$, using the same argument as in (A.2) we have $2^{k} \lesssim \delta(Z)$ and hence

$$
|Z-X| \leq\left|Z-X_{k}\right|+\left|X_{k}-q_{X}\right|+\left|q_{X}-X\right| \leq \frac{1}{c} \delta(Z)+2^{k}+2^{j_{X}+1} \lesssim \delta(Z) .
$$

This proves that $\gamma$ is a good curve for $X$ and $Y$ and completes the case $j_{Y}+2 \leq k$.

Let us finally consider the case $k+1 \leq j_{Y}+2 \leq k+3$. Note that in this situation we clearly have

$$
\left|X_{k}-Y\right| \leq\left|X_{k}-q_{X}\right|+\left|q_{X}-X\right|+|X-Y| \leq 2^{k}+2^{j_{X}+1}+2^{k+1} \leq 2^{k+2} \leq 4|X-Y|
$$

and

$$
\min \left\{\delta\left(X_{k}\right), \delta(Y)\right\} \geq c 2^{k} .
$$

Applying Lemma A.1 there exists a good curve $\gamma_{k}$ joining $X_{k}$ and $Y$. Let $\gamma=$ $\gamma_{X} \cup \gamma_{k}$, and note that $\gamma$ joins $X$ and $Y$. Moreover, since $2^{k} \leq|X-Y|<2^{k+1}$ we have that

$$
\ell(\gamma) \leq \ell\left(\gamma_{X}\right)+\ell\left(\gamma_{k}\right) \lesssim|X-Y|+\left|X_{k}-Y\right| \lesssim|X-Y| .
$$

For $Z \in \gamma_{X}$, (A.3) yields $\delta(Z) \geq c \operatorname{dist}(Z,\{X, Y\})$. If $Z \in \gamma_{k}$ and $\operatorname{dist}\left(Z,\left\{X_{k}, Y\right\}\right)=$ $|Z-Y|$ we obtain that $c|Z-Y| \leq \delta(Z)$ from the construction of $\gamma_{k}$. On the other hand, if $\operatorname{dist}\left(Z,\left\{X_{k}, Y\right\}\right)=\left|X_{k}-Z\right|$, using the same argument as in (A.2) we have $2^{k} \lesssim \delta(Z)$ and hence

$$
|Z-X| \leq\left|Z-X_{k}\right|+\left|X_{k}-q_{X}\right|+\left|q_{X}-X\right| \leq \frac{1}{c} \delta(Z)+2^{k}+2^{j_{X}+1} \lesssim \delta(Z) .
$$

This proves that $\gamma$ is a good curve for $X$ and $Y$ and concludes the proof of Theorem 2.15 .

\section{REFERENCES}

[AS] J. Azzam and R. Schul, Hard Sard: quantitative implicit function and extension theorems for Lipschitz maps, Geom. Funct. Anal. 22 (2012), 1062-1123.

[Bad] M. Badger, Null sets of harmonic measure on NTA domains: Lipschitz approximation revisited, Math. Z. 270 (2012), no. 1-2, 241-262.

[BL] B. Bennewitz and J.L. Lewis, On weak reverse Hölder inequalities for nondoubling harmonic measures, Complex Var. Theory Appl. 49 (2004), no. 7-9, 571-582.

[BS] J. Björn and N. Shanmmugalingam, Poincaré inequalities, uniform domains and extension properties for Newton-Sobolev functions in metric spaces, J. Math. Anal. Appl. 332 (2007), 190-208.

[Chr] M. Christ, A $T(b)$ theorem with remarks on analytic capacity and the Cauchy integral, Colloq. Math., LX/LXI (1990), 601-628.

[Dah] B. Dahlberg, On the absolute continuity of elliptic measure, Amer. J. Math. 108 (1986), 1119-1138.

[Dav] G. David, Approximation of a Reifenberg-flat set by a smooth surface, preprint (arXiv:1211.3222). 
[DJ] G. David and D. Jerison, Lipschitz approximation to hypersurfaces, harmonic measure, and singular integrals, Indiana Univ. Math. J. 39 (1990), no. 3, 831-845.

[DS1] G. David and S. Semmes, Singular integrals and rectifiable sets in $\mathbb{R}^{n}:$ Au-dela des graphes lipschitziens, Asterisque 193 (1991).

[DS2] G. David and S. Semmes, Analysis of and on Uniformly Rectifiable Sets, Mathematical Monographs and Surveys 38, AMS 1993

[GO] F.W. Gehring and B.G. Osgood, Uniform domains and the quasihyperbolic metric, Journal d'Analyse Mathématique 36 (1979), 50-74.

[HM] S. Hofmann and J.M. Martell, Uniform Rectifiability and Harmonic Measure I: Uniform rectifiability implies Poisson kernels in $L^{p}$, to appear, Ann. Sci. École Norm. Sup..

[HMM] S. Hofmann, J.M. Martell and S. Mayboroda, Uniform Rectifiability and Harmonic Measure III: Riesz transform bounds imply uniform rectifiability of boundaries of 1-sided NTA domains, Int. Math. Res. Not. 2014, no. 10, 2702-2729.

[HMU] S. Hofmann, J.M. Martell and I. Uriarte-Tuero, Uniform rectifiability and harmonic measure II: Poisson kernels in $L^{p}$ imply uniform rectifiability, to appear, Duke Math. J.

[HMMM] S. Hofmann, D. Mitrea, M. Mitrea, A. Morris, $L^{p}$-Square Function Estimates on Spaces of Homogeneous Type and on Uniformly Rectifiable Sets, preprint (arXiv:1301.4943).

[JK] D. Jerison and C. Kenig, Boundary behavior of harmonic functions in nontangentially accessible domains, Adv. in Math. 46 (1982), no. 1, 80-147.

[Jo1] P.W. Jones, Square functions, Cauchy integrals, analytic capacity, and harmonic measure, Harmonic analysis and partial differential equations (El Escorial, 1987), 24-68, Lecture Notes in Math., 1384, Springer, Berlin, 1989.

[Jo2] P.W Jones, Extension theorems for BMO, Indiana Univ. Math. J. 29 (1980), 41-66.

[Lav] M. Lavrentiev, Boundary problems in the theory of univalent functions (Russian), Math Sb. 43 (1936), 815-846; AMS Transl. Series 32 (1963), 1-35.

[LN] J.L. Lewis and K. Nyström, Regularity and free boundary regularity for the $p$-Laplace operator in Reifenberg flat and Ahlfors regular domains, Journal Amer. Math. Soc. 25 (2012), 827-862.

[NToV] F. Nazarov, X. Tolsa, and A. Volberg, On the uniform rectifiability of ad-regular measures with bounded Riesz transform operator: The case of codimension 1, preprint (arXiv:1212.5229).

[RR] F. Riesz and M. Riesz, Über die randwerte einer analytischen funktion, Compte Rendues du Quatrième Congrès des Mathématiciens Scandinaves, Stockholm 1916, Almqvists and Wilksels, Upsala, 1920.

[Sem] S. Semmes, Analysis vs. geometry on a class of rectifiable hypersurfaces in $R^{n}$, Indiana Univ. Math. J. 39 (1990), 1005-1035.

[Väi] J. Väisälä, Uniform domains, Tohoku Math. J. (2) 40 (1988), 101-118.

Jonas Azzam, University of Washington, Department of Mathematics, Seattle, WA 98195-4350, USA

E-mail address: jazzam@math.washington.edu

Steve Hofmann, Department of Mathematics, University of Missouri, Columbia, MO 65211, USA

E-mail address: hofmanns@missouri .edu

José María Martell, Instituto de Ciencias Matemáticas CSiC-UAM-UC3M-UCM, Consejo Superior de Investigaciones Científicas, C/ Nicolás Cabrera, 13-15, E-28049 Madrid, Spain

E-mail address: chema.martell@icmat.es 
Kaj Nyström, Department of Mathematics, Uppsala University, S-751 06 Uppsala, Sweden

E-mail address: kaj.nystrom@math.uu.se

Tatiana Toro, University of Washington, Department of Mathematics, Seattle, WA 981954350, USA

E-mail address: toro@math. washington. edu 